\title{
Three-dimensional Architecture of Hair-bundle Linkages Revealed by Electron-microscopic Tomography
}

\author{
Manfred Auer, ${ }^{1,2,3}$ Abrahram J. Koster, ${ }^{4,7}$ Ulrike Ziese, ${ }^{4}$ Chandrajit Bajaj, ${ }^{5}$ Niels Volkmann, ${ }^{6}$ \\ Da Neng Wang, ${ }^{3}$ and A. J. Hudspeth ${ }^{2}$ \\ ${ }^{1}$ Life Sciences Division, Lawrence Berkeley National Laboratory, 1 Cyclotron Road, Berkeley, CA 94720, USA \\ ${ }^{2}$ Howard Hughes Medical Institute and Laboratory of Sensory Neuroscience, The Rockefeller University, Box 314, 1230 York \\ Avenue, New York, NY 10065-6399, USA \\ ${ }^{3}$ Skirball Institute of Biomolecular Medicine, New York University Medical Center, New York, NY 10016, USA \\ ${ }^{4}$ Molecular Cell Biology, Utrecht University, 3584 CH, Utrecht, The Netherlands \\ ${ }^{5}$ Center for Computational Visualization, University of Texas-Austin, Austin, TX 78712, USA \\ ${ }^{6}$ The Burnham Institute, La Jolla, CA 92037, USA
}

Received: 28 November 2007; Accepted: 23 January 2008; Online publication: 18 April 2008

\begin{abstract}
The senses of hearing and balance rest upon mechanoelectrical transduction by the hair bundles of hair cells in the inner ear. Located at the apical cellular surface, each hair bundle comprises several tens of stereocilia and a single kinocilium that are interconnected by extracellular proteinaceous links. Using electron-microscopic tomography of bullfrog saccular sensory epithelia, we examined the three-dimensional structures of basal links, kinociliary links, and tip links. We observed significant differences in the appearances and dimensions of these three structures and found two distinct populations of tip links suggestive of the involvement of different proteins, splice variants, or protein-protein interactions. We noted auxiliary links connecting the upper portions of tip links to the taller stereocilia. Tip links and auxiliary links show a tendency to adopt a globular conformation when disconnected from the membrane surface.
\end{abstract}

Present address: Abrahram J. Koster, Department of Molecular Cell Biology, Leiden University Medical Center, 2300 RC, Leiden, The Netherlands.

Correspondence to: A. J. Hudspeth - Howard Hughes Medical Institute and Laboratory of Sensory Neuroscience $\cdot$ The Rockefeller University · Box 314, 1230 York Avenue, New York, NY 10065-6399, USA. Telephone: +1-212-3277351; fax: +1-212-3277352; email: hudspaj@ rockefeller.edu
Keywords: ankle link, auditory system, basal link, kinociliary link, kinocilium, stereocilium, tip link, vestibular system

\section{INTRODUCTION}

The fundamental role of the hair cell, the sensory receptor of the ear and lateral-line system, is mechanoreception. Key to the hair cell's operation are the filamentous extracellular connections between the processes of the mechanoreceptive hair bundle, of which three types occur in most instances.

First, the hexagonal arrangement of stereocilia is emphasized by links interconnecting them along all three axes (Bägger-Sjöback 1974; Csukas et al. 1987). Because these links impinge upon each stereocilium at the base of its cylindrical shaft, just above its basal taper, they are termed basal links. These connections are acutely dispensable for mechanotransduction (Jacobs and Hudspeth 1990). Connections of a similar form, termed ankle links, occur transiently during the development of mammalian cochlear hair cells. Among several proteins whose deficiency produces Usher Syndrome, three have been proposed to constitute the ankle links: cadherin 23 (Di Palma et al. 2001; Boeda et al. 2002; Siemens et al. 2002), the very large G-proteincoupled receptor, Vlgrlb (McGee et al. 2006), and usherin (Adato et al. 2005; van Wijk et al. 2006). 
In most receptor organs of the inner ear and lateralline system, mechanical stimuli representing sounds, accelerations, or water movements are initially conveyed to the kinocilium by an extracellular accessory structure. Connections of the second type, kinociliary links, then transmit these stimuli to the five longest stereocilia (Hillman and Lewis 1971). Fourier analysis of electron-microscopic images suggests that kinociliary links constitute an array of several dozen fine strands that are arranged in a coiled-coil conformation (Csukas et al. 1987; Tsuprun et al. 2004).

Finally, tip links run obliquely from the ends of stereocilia to the sides of the longest adjacent stereocilia (Pickles et al. 1984). These filaments are probably connected to mechanically sensitive channels and are therefore essential to mechanoelectrical transduction. If exposed to lanthanoid ions or $\mathrm{Ca}^{2+}$ chelators, tip links disappear concomitantly with the loss of responsiveness; as they regenerate over several hours, transduction recovers (Zhao et al. 1996). When analyzed by high-resolution scanning electron microscopy, each tip link appears to comprise two filaments arranged in a right-handed, coiled-coil configuration (Kachar et al. 2000). Fourier analysis of projection images suggests instead a double- or triple-helical structure (Tsuprun and Santi 2000; Tsuprun et al. 2004). A tip link may branch $10-50 \mathrm{~nm}$ below its upper insertion and may contact the membrane at two or three sites at its lower end (Furness and Hackney 1985; Kachar et al. 2000). The tip link may consist of glycosylated protein, for polycationic compounds label it (Neugebauer and Thurm 1987; Tsuprun and Santi 2000), and in avian preparations, it is severed by proteases only after deglycosylation (Kachar et al. 2000). Genetic and immunological evidence localizes cadherin 23 to the tip link (Siemens et al. 2004; Sollner et al. 2004), where it evidently constitutes the structure's upper two thirds (Kazmierczak et al. 2007). The balance of the link is thought to consist of protocadherin 15 (Ahmed et al. 2006).

To gain further insight into the molecular structures of the three classes of links, we conducted electronmicroscopic tomography on hair bundles from the frog's sacculus.

\section{MATERIALS AND METHODS}

\section{Sample preparation}

Bullfrogs were killed, and the saccular maculae were dissected into oxygenated standard saline solution containing of $110 \mathrm{mM} \mathrm{Na}{ }^{+}, 2 \mathrm{mM} \mathrm{K}+4 \mathrm{mM} \mathrm{Ca}^{2+}$, $118 \mathrm{mM} \mathrm{Cl}^{-}, 3 \mathrm{mM}$ D-glucose, and $5 \mathrm{mM} \mathrm{4-(2-}$ hydroxyethyl)-1-piperazineethanesulfonic acid at $\mathrm{pH}$ 7.3. Three distinct sample preparation procedures were employed. The principal differences were whether a detergent was used for controlled extraction before fixation and whether samples were dehydrated on ice or by the method of progressive lowering of temperature (Armbruster et al. 1982; Carlemalm et al. 1982).

In the case of detergent extraction, the sensory epithelia were exposed before fixation for $20 \mathrm{~min}$ on ice to $1 \%$ Triton X-100 in a solution containing $100 \mathrm{mM} \mathrm{KCl}$, $5 \mathrm{mM} \mathrm{MgCl}_{2}, 5 \mathrm{mM}$ ethylene glycol tetraacetic acid, and protease inhibitors. The preparations were then rinsed three times for 2 min each in the solution without detergent.

Sensory epithelia were fixed for $2 \mathrm{~h}$ at room temperature in $3 \%$ glutaraldehyde, $0.2 \%$ tannic acid, $100 \mathrm{mM}$ $\mathrm{KCl}, 5 \mathrm{mM} \mathrm{MgCl}_{2}$, and $20 \mathrm{mM}$ 3-(N-morpholino) propanesulfonic acid at $\mathrm{pH} 6.8$, then rinsed three times for 5 min each at room temperature in the same solution without the glutaraldehyde and tannic acid. After three additional rinses at $4^{\circ} \mathrm{C}$, samples were incubated on ice in $1 \% \mathrm{OsO}_{4}, 10 \mathrm{mM} \mathrm{MgCl}_{2}$, and $100 \mathrm{mM} \mathrm{K} 2 \mathrm{HPO}_{4} / \mathrm{NaH}_{2} \mathrm{PO}_{4}$ at $\mathrm{pH}$ 6.1. After three additional rinses of 5 min each in distilled water at $4^{\circ} \mathrm{C}$, the maculae were stained overnight at $4^{\circ} \mathrm{C}$ in aqueous $1 \%$ uranyl acetate (Taylor et al. 1999).

Conventional dehydration was conducted with ethanol concentrations of $30 \%, 50 \%, 70 \%, 95 \%$, and three rounds of $100 \%$ on ice for 5 min each. For dehydration with progressive lowering of temperature, each incubation period was $20 \mathrm{~min}$, with exposure to $30 \%$ ethanol at $4^{\circ} \mathrm{C}$, to $50 \%$ ethanol at $-20^{\circ} \mathrm{C}$, and to $70 \%$, $95 \%$, and $100 \%$ ethanol at $-35^{\circ} \mathrm{C}$. The tissue was restored to room temperature in $100 \%$ ethanol before embedding in an Epon-Araldite mixture.

After the plastic had been polymerized in a vacuum oven for 3 days, initially at $60^{\circ} \mathrm{C}$ and subsequently at $85^{\circ} \mathrm{C}$, sections were cut at thicknesses of $100-120 \mathrm{~nm}$, placed on $100 \times 400$-mesh slot grids or 200-mesh hexagonal grids, decorated with gold fiducial markers, and coated with a thin layer of carbon.

\section{Data collection and analysis}

Sections were imaged on a Technai $20 \mathrm{LaB}_{6}$, Philips CM200FEG, or Philips EM430 transmission electron microscope. Tilt series were recorded at $1^{\circ}$ intervals for angles of up to $\pm 75^{\circ}$ (Table 1). Projections were aligned and reconstructed into a three-dimensional volume with the software package EM3D (Harlow et al. 2001), PRIISM (Chen et al. 1996), or IMOD (Kremer et al. 1996).

\section{RESULTS}

Improved preservation through low-temperature sample processing

We initially prepared specimens by conventional techniques conducted at room temperature. To improve the preservation of actin filaments, we evaluated several 


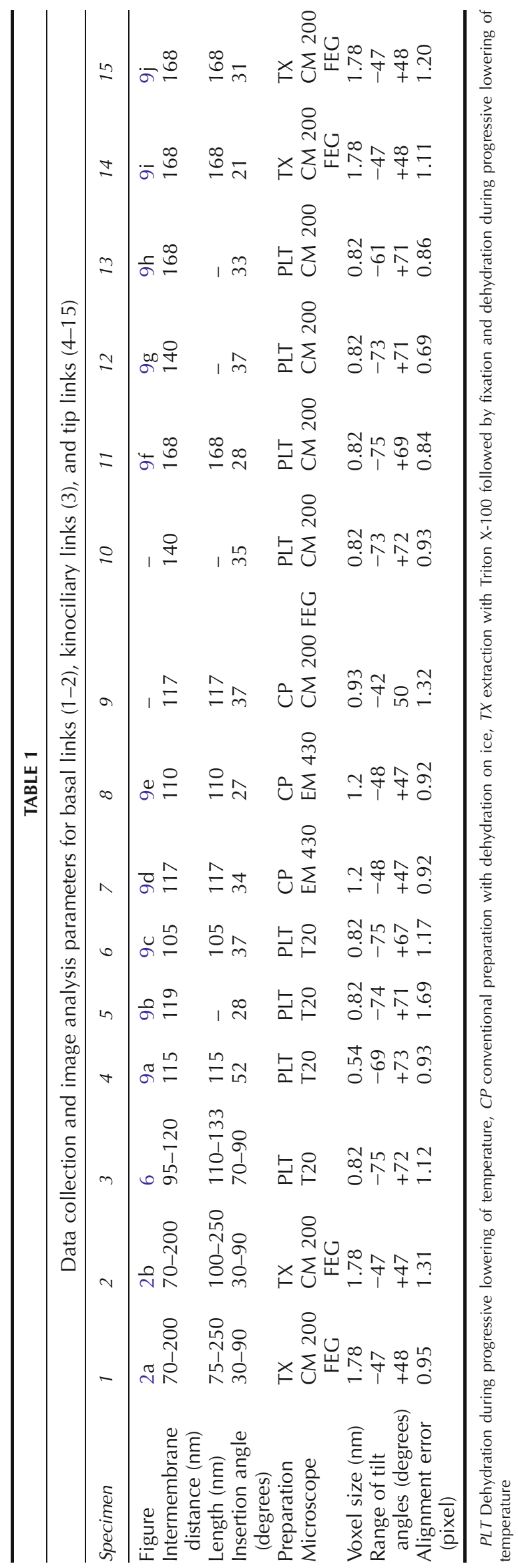

alternative procedures. Preservation was enhanced by conducting the osmium tetroxide postfixation, uranyl acetate staining, and ethanol dehydration steps on ice and by omitting exposure to propylene oxide altogether. The best results were obtained by dehydration during progressive lowering of temperature (Armbruster et al. 1982; Carlemalm et al. 1982).

Two-dimensional projection analysis of hair-bundle links

We examined more than 1,700 pairs of apposed stereocilia that had been prepared by three different procedures. Sections from conventionally processed samples dehydrated at room temperature yielded tip links in only 5 of 97 pairs of opposed stereocilia. In projection images, the apparent lengths of tip links ranged from 75 to $150 \mathrm{~nm}$. Because the overall preservation of these samples was inadequate, however, we did not conduct detailed tomographic analysis.

Using conventional protocols in combination with dehydration on ice, we found in the distal positions of 1,133 stereociliary pairs 181 so oriented as to potentially display tip links. We encountered only 16 tip links, three of which measured $100-110 \mathrm{~nm}$ in projection images, whereas the remainder displayed lengths of 150-170 nm. We enjoyed greater success with dehydration during progressive lowering of temperature. Of 422 stereociliary pairs imaged, 226 were in a favorable orientation; we found 58 tip links, with about one third measuring $110 \mathrm{~nm}$ and the remainder 150$170 \mathrm{~nm}$. We also imaged 30 regions holding kinociliary links. These structures typically measured $110-120 \mathrm{~nm}$ in length but on occasions reached $170 \mathrm{~nm}$. Finally, we encountered in projection images five areas containing 60 well-preserved basal links, whose apparent lengths ranged from 70 to $250 \mathrm{~nm}$.

Three-dimensional tomographic analysis of hair-bundle links

Because it is difficult to infer the exact positions and lengths of sinuous structures in 100 -nm sections, we performed electron-microscopic tomography. We recorded 15 tomographic data sets and conducted reconstructions at voxel sizes ranging from 0.54 to $1.74 \mathrm{~nm}$, with most of the data analyzed at a voxel size of $0.82 \mathrm{~nm}$ (Table 1 ). We deliberately oversampled the data to reduce the risk of substantial distortion of subcellular features during the postprocessing steps of filtering and segmentation.

For interactive exploration of the three-dimensional volumes, we routinely used the Volume Rover package (Bajaj et al. 2003), usually followed by one round of bilateral filtering (Jiang et al. 2003) or denoising by nonlinear anisotropic diffusion (Frangakis and Hegerl 
2001). In some cases, features were extracted by the boundary segmentation approach (Bajaj et al. 2003; Bajaj and $\mathrm{Yu} 2005$ ) or the watershed immersion algorithm (Volkmann 2002). In other instances, bilaterally filtered or unfiltered data were used for interactive segmentation and model building with the Volume Path Tracer and Zone tools in Chimera (Pettersen et al. 2004). Manual, interactive, three-dimensional segmentation required the subjective choice of contour levels; we usually selected the highest threshold that nevertheless ensured connectivity of the features. Depending on the exact contour level chosen, the estimates of filament length and intermembrane distance changed by no more than 3-5 nm. Length and volume measurements were also conducted with Chimera using the Pick Surface Pieces tool. At a chosen contour level, we could determine filament lengths with an accuracy estimated at $2 \mathrm{~nm}$.

\section{Basal links}

We collected two data sets of basal links from hair cells whose membranes had been detergent-treated for reasons unrelated to the present study. The membrane-to-membrane distance spanned by the links ranged in both data sets from 70 to $200 \mathrm{~nm}$ (Fig. 1). Upon tracing 21 basal links in three dimensions, we found lengths ranging from 75 to $250 \mathrm{~nm}$ (Fig. 2). Most filaments did not span the intermembrane distance in the shortest way possible but were curved or S-shaped and inserted on the stereociliary membranes at angles of $60-80^{\circ}$. Filaments of differing length could readily be distinguished in a slice of the reconstructed volume (Fig. 1). Although the voxel volume was limited to $1.74 \mathrm{~nm}$, we estimate that length measurements were accurate within $5 \mathrm{~nm}$.
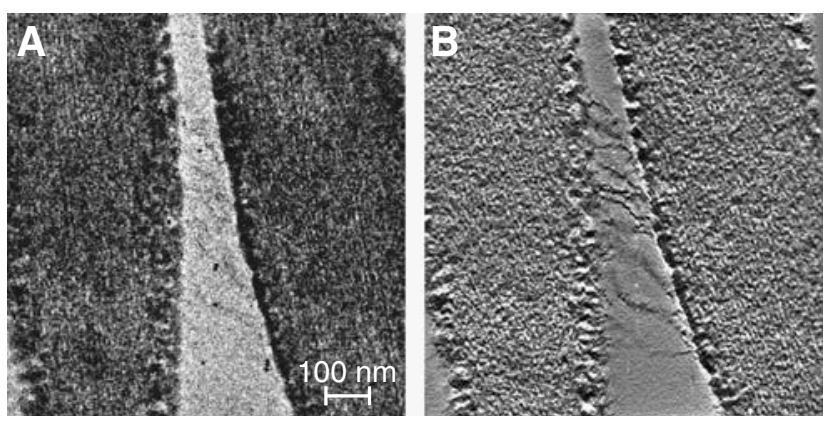

FIG. 1. A A projection view of the basal tapers of two stereocilia in a 100-nm-thick section of a hair bundle extracted with Triton X-100 shows the basal links connecting adjacent stereociliary membranes. In this image as well as those of Figures 5 and 8, the dense colloidal gold particles on the section's surface serve as fiducial markers for the alignment of images in the tilt series. B A 1.7-nm slice through the three-dimensional reconstruction of the tomogram reveals substantially more detail than does the projection image.

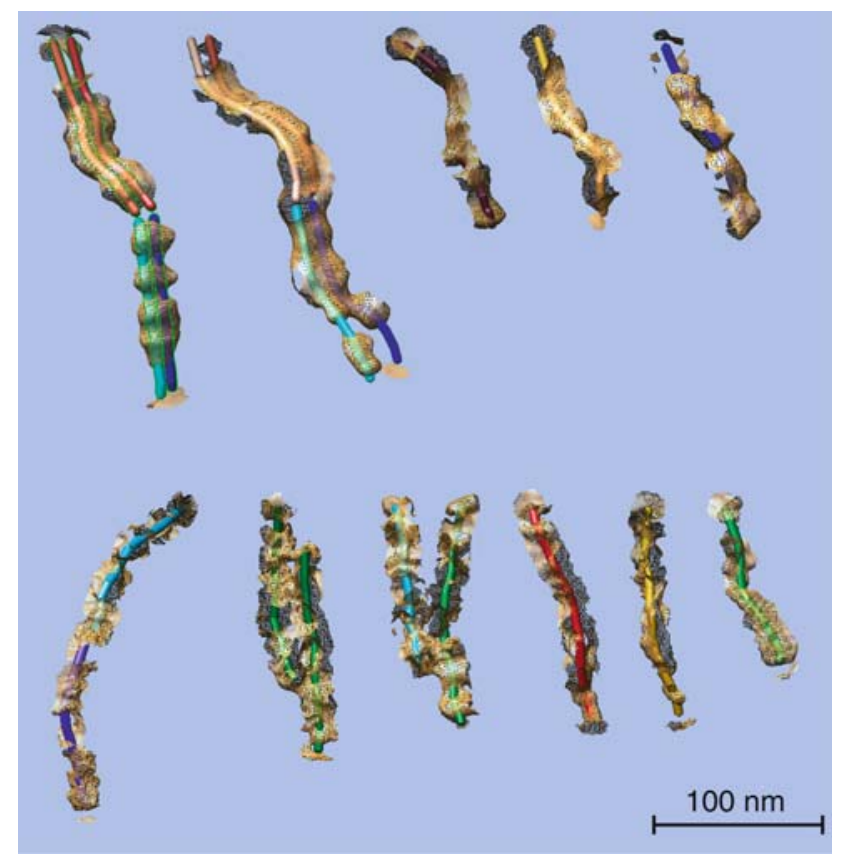

FIG. 2. In these surface renderings of basal links from two data sets, a tube model was interactively traced into the three-dimensional density maps to allow accurate length measurements. The substituents of the longest filaments are about twice the length of the shortest. Some links interact side-by-side as dimers, whereas others may connect to a cell-surface receptor.

Reconstruction of 11 representative basal links showed considerable heterogeneity in their structure (Fig. 3). The length distribution of the filaments suggested distinct classes of filaments, most of which are 120-170 $\mathrm{nm}$ in length, but some of which reach a length of $250 \mathrm{~nm}$ (Fig. 4A; Table 1). The shortest links appear to consist of straight, single filaments $100 \mathrm{~nm}$ in length. Other links seem to consist of single filaments or dimers that are joined either end-to-end or side-by-side. Depending on their thickness, we interpreted the 250-nm-long filaments as either dimers or tetramers. In the instance of the putative tetramers, en face views revealed a doubling of the width of the density compared to other basal-link filaments, suggesting a side-by-side arrangement of two filaments. The maximal number of filaments in intimate lateral contact with one another appeared to be four. Other links consisted of single filaments or dimers that are joined either end-to-end or side-by-side. It therefore appears that multiple proteins of at least two different lengths are involved in basal links.

\section{Kinociliary links}

In a three-dimensional reconstruction of a 100-nm section, we traced 77 kinociliary links, of which 61 spanned the entire distance between the membranes; the remainder left the volume under study or terminated 

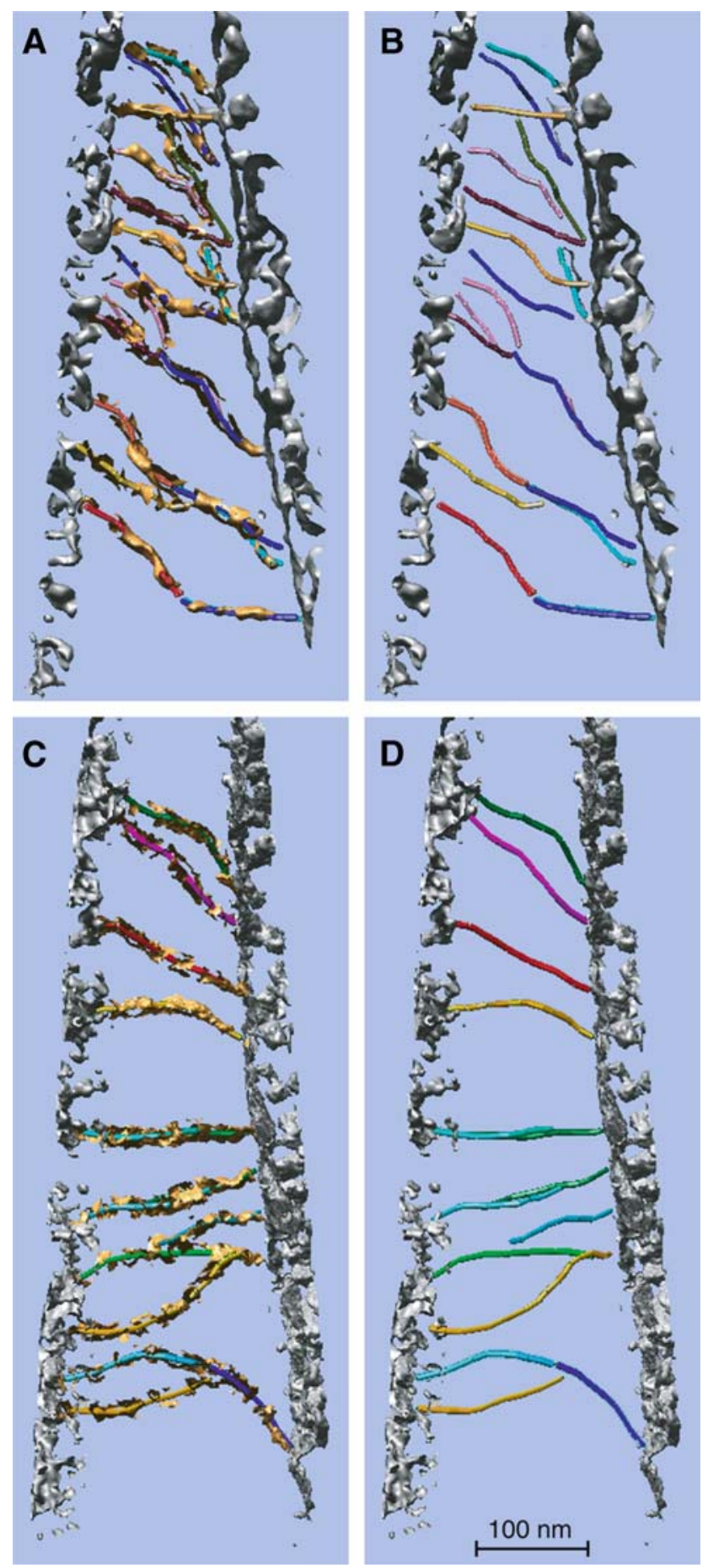

FIG. 3. A A surface rendering of several basal links shows their insertions onto the membrane surface (silver). B Backbone tracings of the same links reveals that, as a consequence of the stereociliary tapers, they extend distances ranging from 70 to $200 \mathrm{~nm}$. C A second data set shows a similar array of surface-rendered basal links. D Note in a tube presentation of these links that the filaments do not connect in the shortest possible way but are instead curved or S-shaped. This behavior suggests a well-defined, rather stiff conformation of the basal-link protein. on other links. The shortest distance between membranes of the tallest stereocilium and its neighboring kinocilium varied from $120 \mathrm{~nm}$ at the proximal end to $95 \mathrm{~nm}$ at the distal end of the kinociliary bulb. Although in projection views along the direction of the electron beam, the links may appear as double or triple filaments

\section{A Basal links}

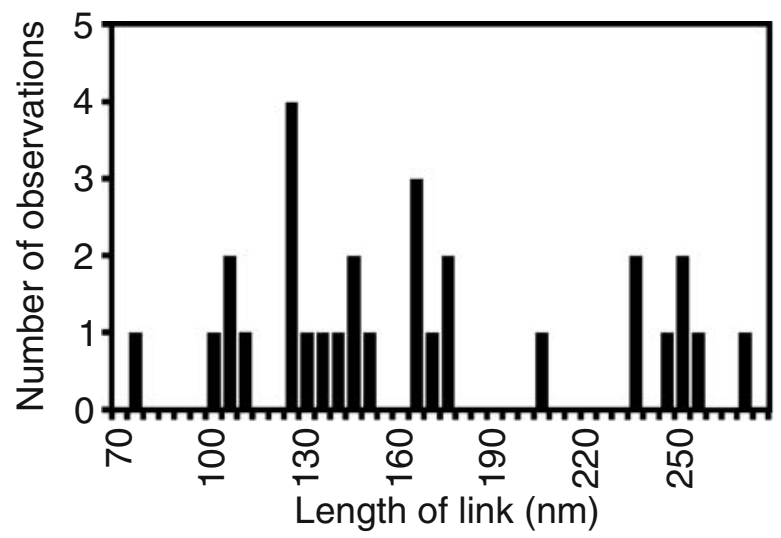

B Kinociliary links

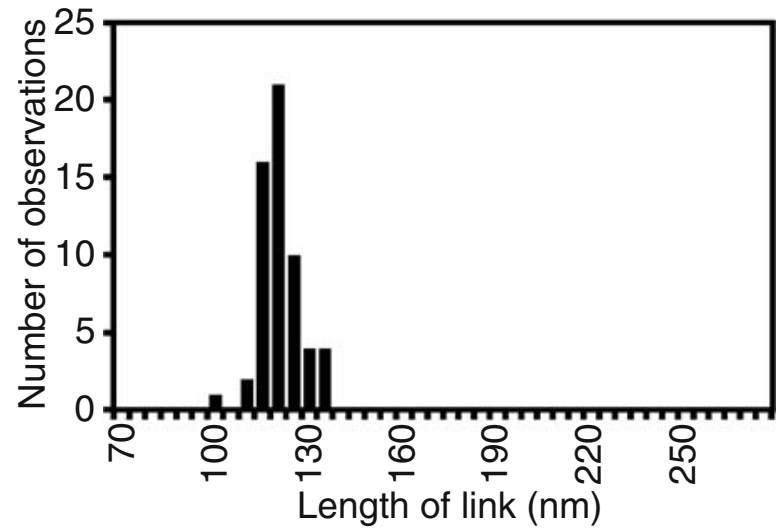

C Tip links

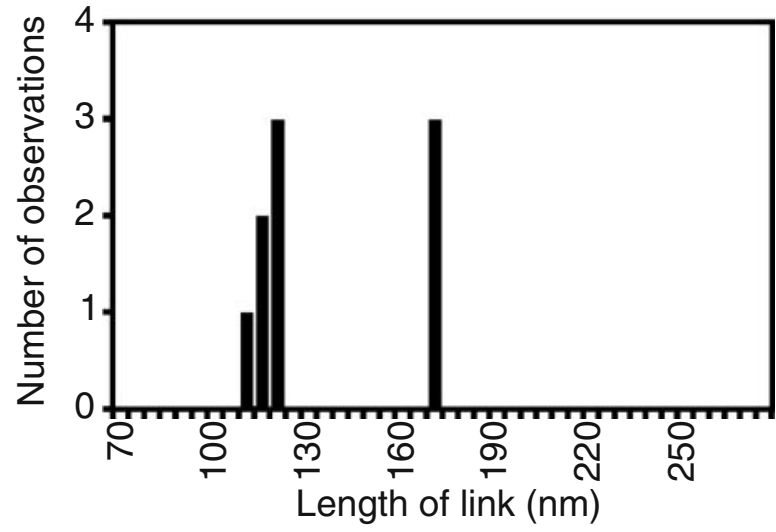

FIG. 4. Histograms display the lengths of different classes of hairbundle link. A Basal links show a very broad distribution of lengths suggestive of at least two structural classes. B Kinociliary links are uniform in dimensions. C Tip links fall into two well-defined length classes. 
twisted around each other (Tsuprun et al. 2004), threedimensional analysis showed that the filaments are separated. Filaments appear to be grouped and insert into only the thicker membrane portions of the kinociliary bulb (Fig. 5). No strict correlation was observed between link length and intermembrane distance; instead, each link was either perpendicular to the membrane or tilted, depending on the length of the filament and the intermembrane distance to be spanned.

Kinociliary links ranged in length from 110 to $133 \mathrm{~nm}$ (Fig. 4B; Table 1). Although most appeared to be straight, some links were slightly bent or S-shaped, occasionally even displaying kinks (Figs. 6 and 7). The apparent thickness of the filaments depended on the contour level selected for surface rendering as well as the degree of filtering for noise reduction, which tends to increase the apparent filament width but is necessary for rendering purposes; based on the density profiles in the unfiltered reconstructions, we estimate the diameter of kinociliary links at $4-5 \mathrm{~nm}$.

\section{Tip links}

We analyzed a total of 12 tip links by electronmicroscopic tomography. Eight of these were intact, with the remaining four disconnected at their basal ends (Figs. 8 and 9). Although some links appeared as two filaments wound helically around each other, others displayed a sheet-like architecture consistent with two filaments lying side-by-side (Fig. 9). Tip links fell into two classes, either 105-120 nm or approximately $170 \mathrm{~nm}$ in length (Fig. 4C). Although we did not measure the lengths of the detached filaments, we also found two populations (Fig. 9). The tip link that
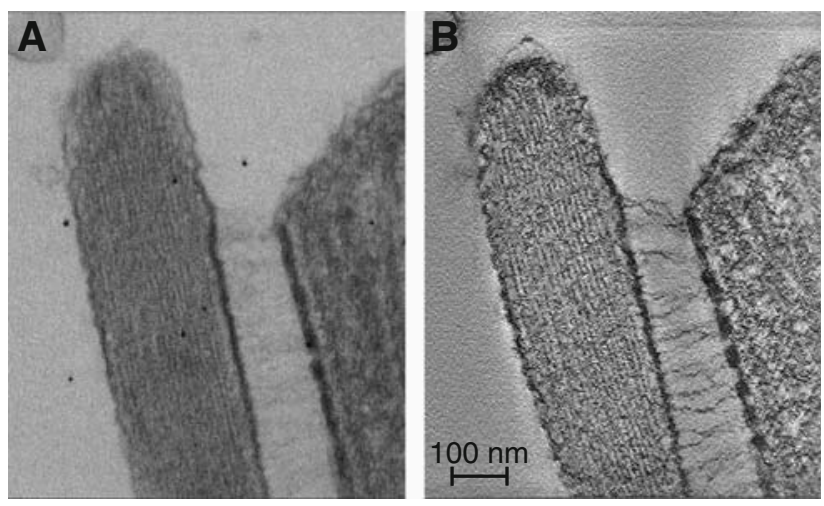

FIG. 5. A A projection view of the top of a hair bundle in a 100-nm-thick section displays the kinociliary links connecting the membrane of the kinocilium (right) with that of an adjacent stereocilium (left). B A 0.82-nm slice through the three-dimensional reconstruction of the corresponding tomogram reveals the wellpreserved actin lattice of the stereocilium and the periodic densities in the kinociliary membrane.

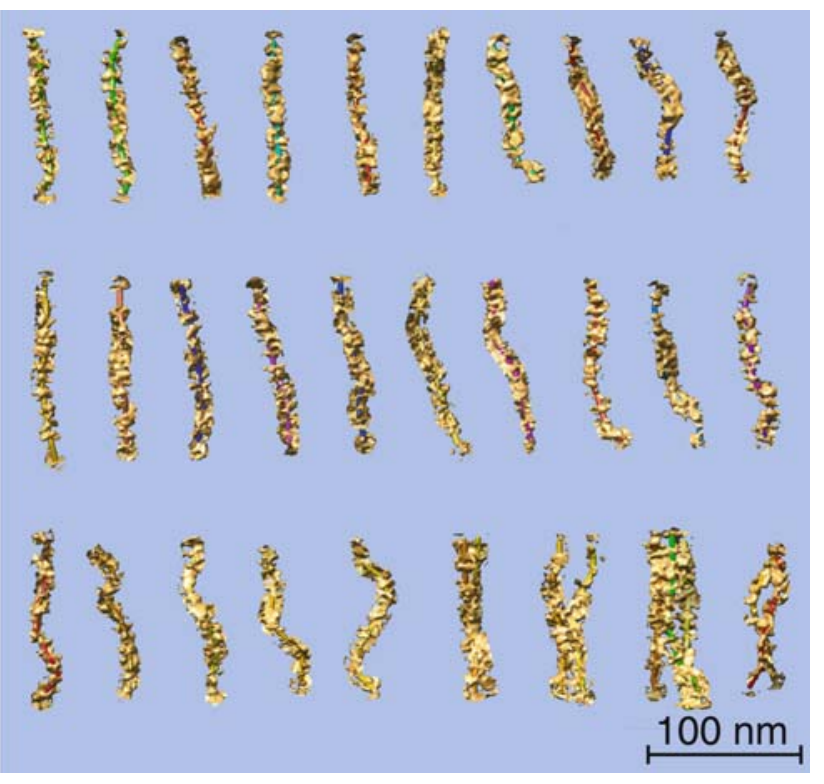

FIG. 6. Kinociliary links represented by volume segmentation are clearly separated from one another and are either straight or slightly curved. Adjacent links occasionally lie too near one another to be resolved.

appeared to be best preserved accorded with a coiledcoil model in which each filament comprised two segments (Fig. 10), with the upper portion containing a longer filament $(93 \mathrm{~nm})$ than the lower portion $(75 \mathrm{~nm})$. A tip link's angle of insertion at the upper stereociliary surface was usually $28-37^{\circ}$ and did not depend on the link's length (Table 1 ).

In at least 6 of the 12 data sets, an additional extracellular density occurred at or above the insertion of

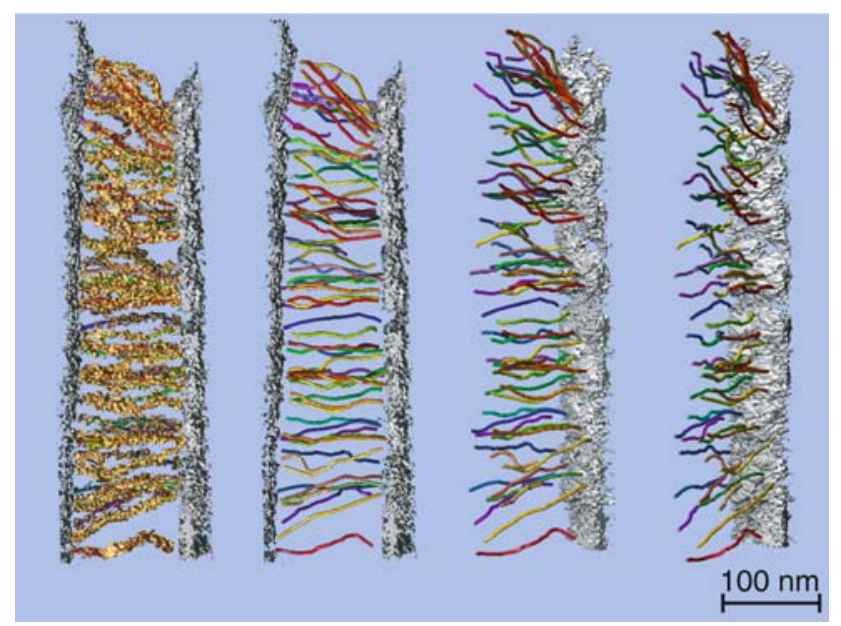

FIG. 7. The surface rendering at the left portrays the kinociliary links between the membranes of a stereocilium (left) and a kinocilium (right). Although the links appear interwound in projection images, progressive rotation of the tube representation reveals their separation. 

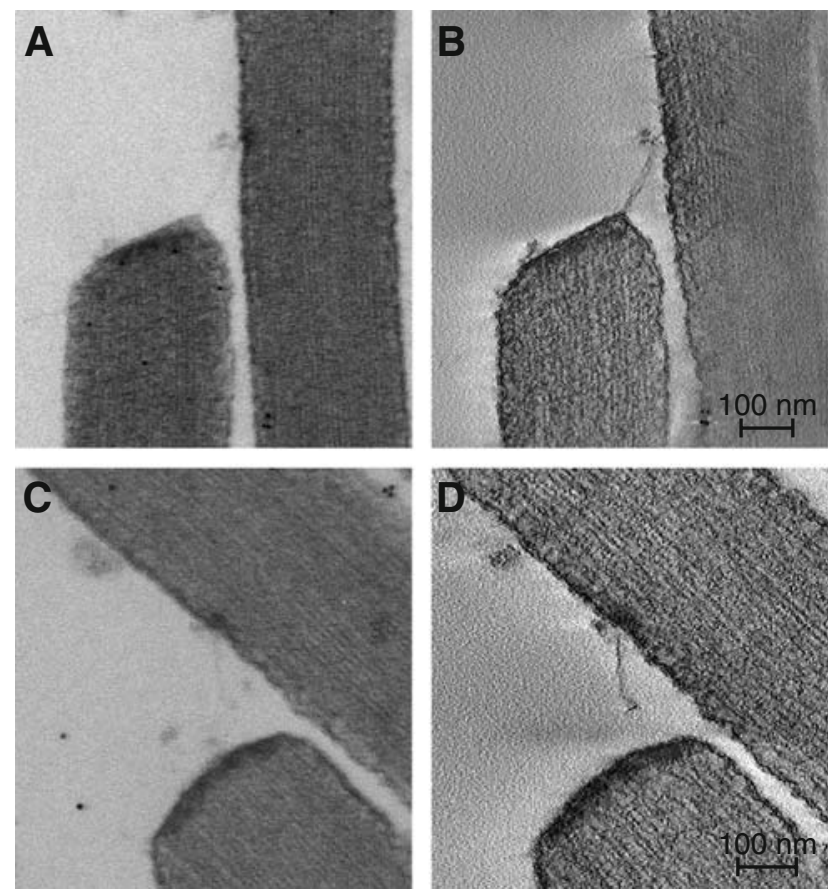

FIG. 8. A A projection view of a 100-nm-thick section of a hair bundle's beveled upper edge shows the tip link connecting the top of one stereocilium with the side of a longer adjacent stereocilium. B A 0.82-nm-thin slice through the three-dimensional reconstruction of the tomogram demonstrates that the tip link is intact and that the membrane of the shorter stereocilium displays tenting. C A projection view of a second specimen shows the well-ordered actin arrays in the stereociliary cytoskeletons. D A 0.82-nm slice through the reconstruction discloses that the tip link is detached from its lower insertion and somewhat curled. Note the tightly coiled densities adjacent to the upper insertions of both tip link and an additional mass $170 \mathrm{~nm}$ above insertion of the main link.

the tip link on the taller stereocilium (Fig. 8). Although these structures appeared amorphous in projection views, three-dimensional visualization resolved them as curled filaments (Fig. 11). We also encountered one instance of two auxiliary links running from the middle of the main link toward the membrane of the taller stereocilium (Fig. 11). At least one of the two links was anchored into the stereociliary surface by an apparent receptor molecule $20 \mathrm{~nm}$ in length. The auxiliary filament contacted the membrane surface 55-60 nm from its main link's attachment site, then turned $90^{\circ}$ and ran perpendicular to the stereociliary axis and parallel to the membrane surface above the main link, where together with the second auxiliary link it formed the lower portion of a bilobed feature. The upper and lower lobes connected at their distal ends; the upper lobe appeared to be larger than the lower. Using the Pick Surface Pieces tool in Chimera (Pettersen et al. 2004), we measured the following approximate volumes: main link $24,400 \mathrm{~nm}^{3}$, auxiliary link $3,100 \mathrm{~nm}^{3}$, lower lobe $7,800 \mathrm{~nm}^{3}$, and upper lobe $11,500 \mathrm{~nm}^{3}$.

\section{DISCUSSION}

\section{Improved sample preservation}

The low probability of finding tip links and the limited success associated with cutting frozen hydrated sections precluded cryo-electron microscopy in the present study. The contrast in our specimens therefore stemmed from the stain surrounding and penetrating proteins rather than from the proteins themselves. However, comparison of the electron density for actin filaments in our specimens with an atomic model derived from X-ray crystallography and cryo-electron microscopy supports the validity of molecular interpretations of our tomograms. Moreover, metal-stained,

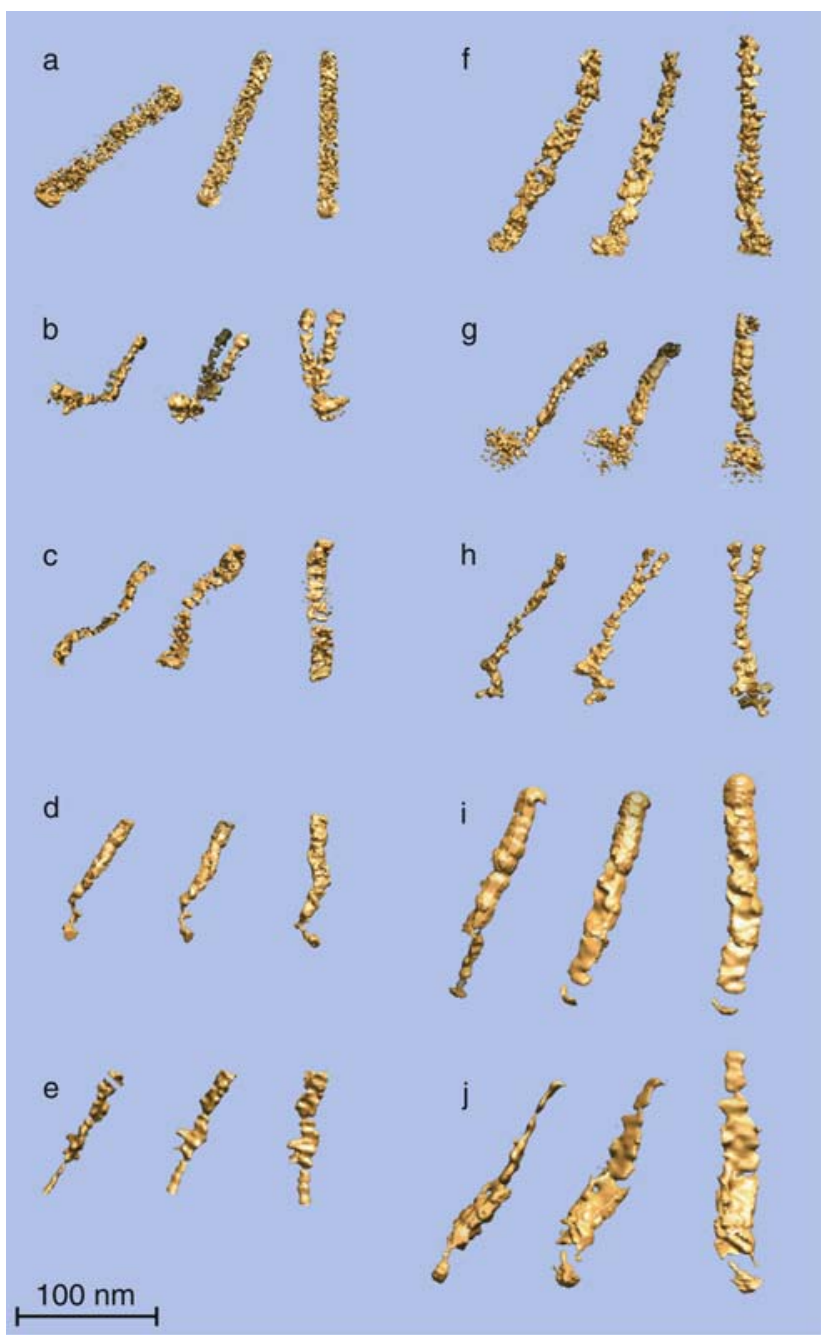

FIG. 9. In a gallery of tip links from 10 of the 12 data sets, each link is shown in three different orientations defined by successive rotations of $45^{\circ}$. For each triplet, the view on the right portrays the tip link as projected onto the plane of the longer stereocilium's membrane surface. Roughly equal numbers of long and short tip links were encountered. The labels identify the images for which Table 1 provides technical information. 


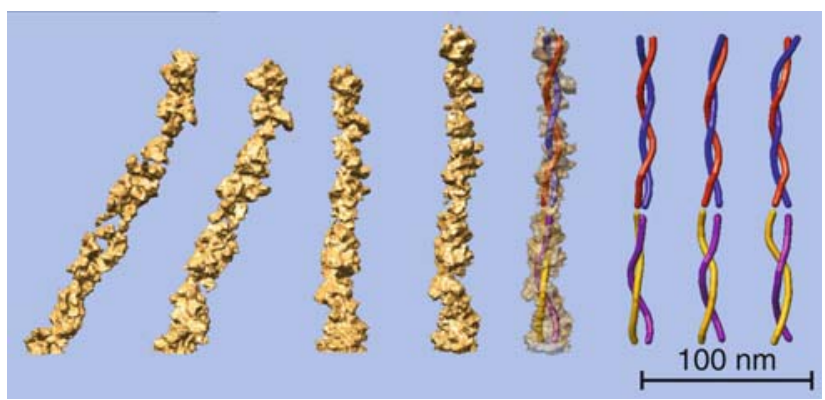

FIG. 10. These higher-magnification views of the best-preserved tip link include a molecular model interactively traced into the threedimensional density. At the left is a view of the link in the orientation typically observed in sectioned hair bundles. The second and third images show the results of successive rotations by $45^{\circ}$; the fourth image reflects both a rotation of $90^{\circ}$ and a tilt of $28^{\circ}$ to place the reconstruction in the plane of the illustration. The fifth image shows an interactively traced model within the tip link; the final three images show the model in the same orientation and after axial rotations of 45 and $90^{\circ}$.

resin-embedded tissue has been successfully interpreted at the molecular level for acto-myosin complexes in insect flight muscle (Taylor et al. 1999) and for cadherin molecules in desmosomes (He et al. 2003). Repeated attempts of high-pressure freezing of frog sensory epithelia were unsuccessful in preserving hair-bundle architecture.

Although we encountered some wrinkling of stereociliary membranes, dehydration with progressive lowering of temperature (Small 1981) resulted in generally good preservation, most clearly visible in the stereociliary cytoskeleton. Because the spacing of actin filaments in stereocilia accords with that measured by electron microscopy of filaments cross-linked by fimbrin (Volkmann et al. 2001), the stereocilia probably did not shrink significantly during preparation (Luther et al. 1988; Braunfeld et al. 1994), and hence differences in filament length cannot be attributed to shrinkage.

\section{Organization of basal links}

Because basal links are S-shaped or smoothly curved and do not span the extracellular space directly, they evidently have a somewhat rigid structure. The density maps for long basal-link filaments are best matched by a tetramer, such as a cadherin (Shapiro et al. 1995; Nagar et al. 1996), comprising two dimers arranged side-by-side and joined head-to-head. Our data are compatible with a tetramer of cadherin 23, which extends $122 \mathrm{~nm}$ for a model including 27 extracellular ectodomains. However, the short isoform of usherin, also predicted to be $125 \mathrm{~nm}$ in length (Adato et al. 2005; van Wijk et al. 2006), would be an equally good candidate. In addition, the extracellular portion of Vlgr1b spans $180 \mathrm{~nm}$ (McMillan and White 2004), approximately the length of a subgroup of the basal links that were found to be $165-175 \mathrm{~nm}$ long.

\section{Organization of kinociliary links}

Unlike basal links, kinociliary links are typically oriented orthogonal to membrane surfaces. The average length of a kinociliary link agrees with the anticipated dimensions of a cadherin 23 molecule: With 27 extracellular domains each $4.5 \mathrm{~nm}$ in length, such a filament would extend about $122 \mathrm{~nm}$. This resemblance may reflect similarities in the molecular identities of the two structures, for a monoclonal antibody that labels tip links also marks kinociliary links (Goodyear and Richardson 2003). Most kinociliary links analyzed by tomography are shorter than expected for a full-length cadherin 23 monomer bound to a surface receptor, although a diminished splice isoform could be accommodated. The identity of the kinociliary links therefore remains uncertain.
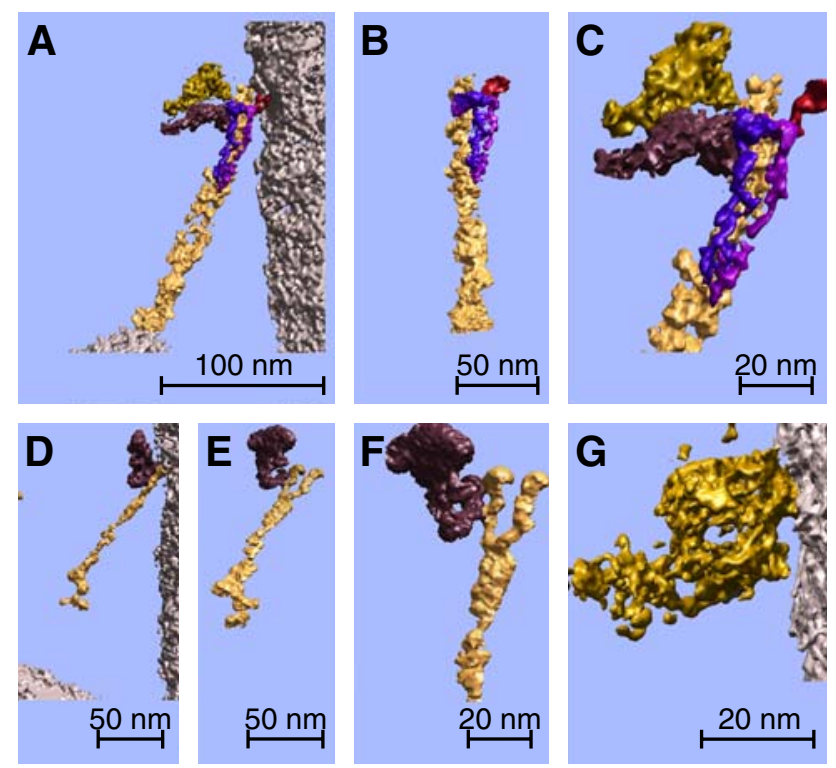

FIG. 11. A This surface rendering of the data set presented in Fig. 8b depicts a segmented tip link interconnecting two stereociliary membranes. The main tip link is shown in gold, two strands connecting to the tip link and membrane are rendered in purple, and a membrane-anchored structure is displayed in red; the stereociliary membranes are portrayed in silver. B The same link is depicted in isolation after a rotation of $90^{\circ}$. $\mathbf{C}$ In a higher-magnification view of the link's upper insertion, the lower component of a bilobed feature is shown in maroon and the upper constituent in green. D Another surface rendering of the data set presented in Figure $8 \mathrm{~d}$ displays a detached tip link. E, F Successively higher-magnification views emphasize the auxiliary link, colored maroon, at the main link's upper end. G Another structure, possibly the remainder of a broken auxiliary link, resides $170 \mathrm{~nm}$ above the tip link's insertion. 


\section{Organization of tip links}

The striking dichotomy that we observed in the lengths of tip links both in two-dimensional projection images and in three-dimensional tomographic reconstructions is also apparent in published images (Zhao et al. 1996; Sollner et al. 2004). Variations in tip-link dimensions cannot be attributed to tissue shrinkage, for in some instances long and short links coexisted in the same hair bundle. We therefore believe that such differences reflect heterogeneity in protein identity, splice isoform, or conformation.

The curled appearance of detached tip links suggests that these structures are not flexurally rigid. Our results do not bear on the extensibility of the links, however, and thus on their possible contribution to the stiffness of the gating springs for mechanoelectrical transduction.

In the best-preserved samples, we found evidence for an auxiliary link that originates from the main tip link. The auxiliary link appears to be anchored by a protruding density to the taller stereocilium's membrane adjacent to the main link's insertion. Although it is unclear whether the auxiliary link and main tip link are made of the same proteins, both structures occupy comparable volumes and curl up when detached. The divergence of an auxiliary link from the main tip link may underlie the forking of tip links (Furness and Hackney 1985; Kachar et al. 2000), for both filaments in the main link connect to the membrane surface. In our projection images, as well as in published micrographs (Zhao et al. 1996; Siemens et al. 2004), the sides of stereocilia often display globular extracellular densities that may reflect detached auxiliary links.

Our results are in agreement with the recent demonstration that the mammalian tip link comprises a head-to-head heterodimer of paired cadherin 23 and protocadherin 15 filaments (Kazmierczak et al. 2007). In particular, this structure would be expected to yield links about $170 \mathrm{~nm}$ in total length, a value that accords with the longer tip links in the present study. A large proportion of the tip links from the bullfrog's sacculus are significantly shorter, however, with an average length of about $115 \mathrm{~nm}$. It is interesting to note that this value is near the expected length of a cadherin 23 molecule: With 27 extracellular domains each $4.5 \mathrm{~nm}$ in length, such a filament would extend about $122 \mathrm{~nm}$. It is therefore possible that some tip links consist simply of parallel dimers of cadherin 23. Two other alternatives may also be considered. First, the shorter tip links might include an alternative splice isoform of cadherin 23, an altogether different cadherin, or a distinct class of protein. A second possibility is that the shorter links stem from an alternative site of binding between cadherin 23 and protocadherin 15 dimers such that the two filaments overlap along nearly the entire extent of the shorter molecules.

\section{ACKNOWLEDGMENTS}

We thank Drs. D. Agard, E. Branlund, M. Braunfeld, T. Goddard, W. Z. He, R. Hegerl, B. Keszthelyi, J. Lyle, F. Macaluso, R. Marshall, R. McIntosh, U. J. McMahan, G. Min, D. Stokes, and K. Taylor for help with data collection and processing. We are particularly indebted to Drs. K. McDonald and M. Reedy for suggestions about sample preservation. Dr. K. Downing and the members of our research groups provided helpful comments on the manuscript. This project was supported by National Institutes of Health grants DC00241, DC07680, EB4873, GM064473, GM073087, GM074258, and RR020647, by National Science Foundation grants ITR-EIA0325550 and CNS-0540033, and by the Director, Office of Science, of the US Department of Energy under contract DEAC03-76SF00098. M. A. was supported by an Agouron Fellowship in Structural Biology from the Jane Coffin Childs Memorial fund and by HFSPO fellowship LT-0532; A. J. H. is an Investigator of Howard Hughes Medical Institute.

\section{REFERENCES}

Adato A, Lefevre G, Delprat B, Michel V, Michalski N, Chardenoux S, Weil D, El-Amraoui A, Petit C. Usherin, the defective protein in Usher syndrome type IIA, is likely to be a component of interstereocilia ankle links in the inner ear sensory cells. Hum. Mol. Genet. 14:3921-3932, 2005.

Ahmed ZM, Goodyear R, Riazuddin S, Lagziel A, Legan PK, Behra M, Burgess SM, Lilley KS, Wilcox ER, Riazudddin S, Griffith AJ, Frolenkov GI, Belyantseva IA, Richardson GP, Friedman TB. The tip-link antigen, a protein associated with the transduction complex of sensory hair cells, is protocadherin-15. J. Neurosci. 26:7022-7034, 2006

armbruster BL, Carlemalm E, Chiovetti R, Garavito RM, Нobot Ja, Kellenberger E, Villiger W. Specimen preparation for electron microscopy using low temperature embedding resins. J. Microsc. 126:77-85, 1982.

BAGger-SJöBÄCK D. The sensory hairs and their attachments in the lizard basilar papilla. Brain Behav. Evol. 10:88-94, 1974.

BAJAJ C, YU Z. Geometric processing of reconstructed 3D maps of molecular complexes. In: Aluru S (ed) Handbook of computational molecular biology, computer and information sciences series, part 8. Boca Raton, FL, Chapman \& Hall/CRC, pp. 1-19, 2005.

BajaJ C, Yu Z, Auer M. Volumetric feature extraction and visualization of tomographic molecular imaging. J. Struct. Biol. 144:132143, 2003.

Boeda B, El-Amraoui A, Bahloul A, Goodyear R, Daviet L, Blanchard S, Perfettini I, Fath KR, Shorte S, Reiners J, Houdusse A, Legrain P, Wolfrum U, Richardson G, Petit C. Myosin ViIa, harmonin and cadherin 23, three Usher I gene products that cooperate to shape the sensory hair cell bundle. Embo J. 21:6689-6699, 2002

Braunfeld MB, Koster AJ, Sedat JW, Agard DA. Cryo automated electron tomography: towards high-resolution reconstructions of plastic-embedded structures. J. Microsc. 174:75-84, 1994.

Carlemalm E, Armbruster BL, Chiovetti R, Garavito RM, Hobot JA, Villiger W, Kellenberger E. Perspectives for achieving improved information by the observation of thin sections in the electron microscope. Tokai J. Exp. Clin. Med. 7(Suppl):33-42, 1982.

Chen H, Hughes DD, Chan TA, Sedat JW, Agard. IVE (Image Visualization Environment): a software platform for all threedimensional microscopy applications. J. Struct. Biol. 116:56-60, 1996. 
Csukas SR, Rosenquist TH, Mulroy MJ. Connections between stereocilia in auditory hair cells of the alligator lizard. Hear. Res. 30:147-155, 1987.

Di Palma F, Pellegrino R, Noben-Trauth K. Genomic structure, alternative splice forms and normal and mutant alleles of cadherin 23 (Cdh23). Gene 281:31-41, 2001.

Frangakis AS, Hegerl R. Noise reduction in electron tomographic reconstructions using nonlinear anisotropic diffusion. J. Struct. Biol. 135:239-250, 2001.

Furness DN, HaCkNey CM. Cross-links between stereocilia in the guinea pig cochlea. Hear. Res. 18:177-188, 1985.

GoOdYeAR RJ, Richardson GP. A novel antigen sensitive to calcium chelation that is associated with the tip links and kinocilial links of sensory hair bundles. J. Neurosci. 23:4878-4887, 2003.

Harlow ML, Ress D, Stoschek A, Marshall RM, McMahan UJ. The architecture of active zone material at the frog's neuromuscular junction. Nature 409:479-484, 2001.

He W, Cowin P, Stokes DL. Untangling desmosomal knots with electron tomography. Science 302:109-113, 2003.

Hillman DE, Lewis ER. Morphological basis for a mechanical linkage in otolithic receptor transduction it the frog. Science 174:416-419, 1971.

JacoBs RA, HudSPETh AJ. Ultrastructural correlates of mechanoelectrical transduction in hair cells of the bullfrog's internal ear. Cold Spring Harbor Symp. Quant. Biol. 55:547-561, 1990.

Jiang W, Baker ML, Wu Q Bajaj C, Chiu W. Applications of a bilateral denoising filter in biological electron microscopy. J. Struct. Biol. 144:114-122, 2003.

Kachar B, Parakkal M, Kurc M, Zhao Y, Gillespie PG. Highresolution structure of hair-cell tip links. Proc. Natl. Acad. Sci. USA 97:13336-13341, 2000.

Kazmierczak P, Sakaguchi H, Tokita J, Wilson-Kubalek EM, Milligan RA, Müller U, Kachar B. Cadherin 23 and protocadherin 15 interact to form tip-link filaments in sensory hair cells. Nature 449:87-91, 2007

Kremer JR, Mastronarde DN, McIntosh JR. Computer visualization of three-dimensional image data using IMOD. J. Struct. Biol. 116:71-76, 1996.

LUTHER PK, LAWRENCE MC, CrowTHER RA. A method for monitoring the collapse of plastic sections as a function of electron dose. Ultramicroscopy 24:7-18, 1988.

McGee J, Goodyear RJ, McMillan DR, Stauffer EA, Holt JR, Locke KG, Birch DG, Legan PK, White PC, Walsh E, Richardson GP. The very large G-protein-coupled receptor VLGR1: a component of the ankle link complex required for normal development of auditory hair bundles. J. Neurosci. 26:6543-6553, 2006.

MCMillan DR, White PC. Loss of the transmembrane and cytoplasmic domains of the very large G-protein-coupled receptor-1 (VLGR1 or Mass1) causes audiogenic seizures in mice. Mol. Cell. Neurosci. 26:322-329, 2004.

Nagar B, Overduin M, IKuRa M, Rini JM. Structural basis of calciuminduced E-cadherin rigidification and dimerization. Nature 380:360-364, 1996.
Neugebauer DC, Thurm U. Surface charges of the membrane and cell adhesion substances determine the structural integrity of hair bundles from the inner ear fish. Cell Tissue Res. 249:199207, 1987.

Pettersen EF, Goddard TD, Hung CC, Couch GS, Greenblatt DM, Meng EC, Ferrin TE. UCSF Chimera-a visualization system for exploratory research and analysis. J. Comput. Chem. 25:16051612, 2004.

Pickles JO, Comis SD, Osborne MP. Cross-links between stereocilia in the guinea pig organ of Corti, and their possible relation to sensory transduction. Hear. Res. 15:103-112, 1984.

Shapiro L, Fannon AM, Kwong PD, Thompson A, Lehmann MS, Grubel G, Legrand JF, Als-Nielsen J, Colman DR, Hendrickson WA. Structural basis of cell-cell adhesion by cadherins. Nature 374:327-337, 1995.

Siemens J, Kazmierczak P, Reynolds A, Sticker M, Littlewood-Evans A, MüLler U. The Usher syndrome proteins cadherin 23 and harmonin form a complex by means of PDZ-domain interactions. Proc. Natl. Acad. Sci. USA 99:14946-14951, 2002.

Siemens J, Lillo C, Dumont RA, Reynolds A, Williams DS, Gillespie PG, Muller U. Cadherin 23 is a component of the tip link in hair-cell stereocilia. Nature 428:950-955, 2004.

SMALL JV. Organization of actin in the leading edge of cultured cells: influence of osmium tetroxide and dehydration on the ultrastructure of actin meshworks. J. Cell Biol. 91:695-705, 1981.

Sollner C, Rauch GJ, Siemens J, Geisler R, Schuster SC, Müller U, Nicolson T. Mutations in cadherin 23 affect tip links in zebrafish sensory hair cells. Nature 428:955-959, 2004.

Taylor KA, Schmitz H, Reedy MC, Goldman YE, Franzini-Armstrong C, Sasaki H, Tregear RT, Poole K, Lucaveche C, Edwards RJ, Chen LF, WinkLeR H, REEDy MK. Tomographic 3D reconstruction of quick-frozen, Ca2+-activated contracting insect flight muscle. Cell 99:421-431, 1999.

Tsuprun V, SANTI P. Helical structure of hair cell stereocilia tip links in the chinchilla cochlea. J. Assoc. Res. Otolaryngol. 1:224-231, 2000.

Tsuprun V, GoOdyear RJ, Richardson GP. The structure of tip links and kinocilial links in avian sensory hair bundles. Biophys. J. 87:4106-4112, 2004.

van Wijk E, van der Zwaag B, Peters T, Zimmermann U, Te Brinke H, Kersten FF, Marker T, Aller E, Hoefsloot lH, Cremers CW, Cremers FP, Wolfrum U, Knipper M, Roepman R, Kremer H. The DFNB31 gene product whirlin connects to the Usher protein network in the cochlea and retina by direct association with USH2A and VLGR1. Hum. Mol. Genet. 15:751-765, 2006.

VolKMANN N. A novel three-dimensional variant of the watershed transform for segmentation of electron density maps. J. Struct. Biol. 138:123-129, 2002.

Volkmann N, DeRosier D, Matsudaira P, Hanein D. An atomic model of actin filaments cross-linked by fimbrin and its implications for bundle assembly and function. J. Cell. Biol. 153:947-956, 2001.

Zhaо Y, Yамодн EN, GillesPie PG. Regeneration of broken tip links and restoration of mechanical transduction in hair cells. Proc. Natl. Acad. Sci. USA 93:15469-15474, 1996. 